\title{
Insights into the role of iron in immature rat model of hypoxic-ischemic brain injury
}

\author{
ZI-WEI WANG ${ }^{1}$, LI-JUN YANG ${ }^{1}$, YING-XUE DING $^{1}$, YAN-ZHONG CHANG $^{2}$ and HONG CUI ${ }^{1}$ \\ ${ }^{1}$ Department of Pediatrics, Beijing Friendship Hospital, Capital Medical University, Beijing 100050; \\ ${ }^{2}$ Department of Life Sciences, Hebei Normal University, Shijiazhuang, Hebei 050024, P.R. China
}

Received November 14, 2014; Accepted April 6, 2016

DOI: $10.3892 / \mathrm{etm} .2016 .3550$

\begin{abstract}
This study aimed to investigate the role of iron in the occurrence and development of hypoxic-ischemic brain injury (HIBI) in immature rat models using 3-day-old Sprague Dawley rats. Normal control (NC), hypoxic-ischemic (HI), anemia, HI + ischemia, early iron treatment and late iron treatment groups were established. Rat brain tissue sections were stained with hematoxylin and eosin and pathologically evaluated. Iron content and mRNA expression levels of iron regulatory protein 2 (IRP2) and transferrin receptor in the brain tissues were measured. Ultrastructural changes in the actin, microtubules, myelin and mitochondria of oligodendrocytes and axons were examined by electron microscopy. Numbers of viable myelin sheaths and oligodendrocytes in the periventricular area were also observed. Pathological damage of brain tissue in the HI group was markedly increased compared with that in the NC group. Furthermore, there was a higher iron content and reduced number of viable oligodendrocytes in the periventricular area of the HI group compared with the NC group. No significant difference in iron content was observed between the HI + anemia and NC groups. The number of viable oligodendrocytes in the $\mathrm{HI}+$ anemia group was increased compared with that in the HI group, and the number in the $\mathrm{HI}+$ anemia group with late iron treatment was lower compared with that in the NC group and increased compared with that in the $\mathrm{HI}+$ anemia group. Electron microscopy revealed a significantly higher number of myelin sheaths in the HI + anemia group than in the HI group. IRP2 mRNA expression levels in the brain tissues were significantly decreased in the HI + anemia group compared with the HI group. The results suggest that anemia may reduce the rate
\end{abstract}

Correspondence to: Dr Hong Cui, Department of Pediatrics, Beijing Friendship Hospital, Capital Medical University, 95 Yongan Road, Xuanwu, Beijing 100050, P.R. China

E-mail: cuihong66@yeah.net

Key words: iron, anemia, hypoxic ischemic brain injury, iron regulatory protein, transferrin receptor, premature newborn rat, myelin of increase of iron content of the brain following HI. However, the early occurrence of anemia may protect against HIBI.

\section{Introduction}

The survival rate of extremely premature infants (born at $<28$-weeks of gestation) has been greatly enhanced due to multiple factors, such as technological advances, the use of antenatal steroids, surfactant therapy and improvements in antenatal and postnatal care (1). However, problems remain regarding the prognosis of nervous system injuries and neurological disabilities, including cerebral palsy and other development disorders in premature infants $(2,3)$. Since the underlying mechanisms of brain injury and repair in premature infants are unclear, there has been a lack of progress in the development of clinical treatments for these disorders.

Iron has an important role in vivo due to its association with hemoglobin and oxygen-carrying function. In the brain, iron also acts as an important component of enzyme systems; it is essential for a number of enzymes involved in neurotransmitter synthesis, including tryptophan hydroxylase (serotonin), tyrosine hydroxylase (norepinephrine) and dopamine (4,5).Previous studies have suggested that iron may contribute to nervous system development in children; an iron deficiency during infancy has been shown to affect the development of the nervous system, leading to poor intelligence, a lack of concentration, learning difficulties and various other symptoms $(4,6)$. Conversely, other studies have reported an increased brain iron content in various adult demyelinating diseases, including Alzheimer's disease, Parkinson's disease, Huntington's chorea (7-9) and obstructive cerebral hemorrhage $(10,11)$. Notably, iron chelation therapy, including deferoxamine, is able to markedly alleviate these diseases (12). In addition, it has been hypothesized that iron increases oxidative damage and participates in associated pathways, which may be a potential mechanism (13).

The present study hypothesized that iron deficiency or anemia may protect against the incidence of hypoxic-ischemic brain injury (HIBI) in neonatal or premature infants, provided that the anemia is not severe enough to cause serious complications. Conversely, during the recovery stage of HIBI, the attenuation of anemia may be beneficial, in order to promote the recovery of nerve injury and nervous system development in the future. 
The present study aimed to investigate the effects of anemia in an immature rat model of HIBI. In addition, the therapeutic potential of iron treatment on the rats, and its underlying mechanism, were analyzed. The results of the present study may provide a foundation for future studies and identify novel therapeutic targets for brain injury function rehabilitation in premature children.

\section{Materials and methods}

Experimental design. The present study was conducted at the Experimental Animal Center of Beijing Friendship Hospital, Capital Medical University (Beijing, China), during the period of July 2012 to February 2013. All experimental procedures were approved by the Animal Study Ethics Committee of Beijing Friendship Hospital, and were conducted in accordance with the institutional criteria for the care and use of laboratory animals in research.

Experimental materials. A total of 90 healthy 2-day-old Sprague Dawley (SD) rats (weight, 6-8 g) of both genders were purchased from Beijing Vital River Laboratory Animal Technology Co., Ltd. (Beijing, China). The rats were maintained under a 12 -h light/dark cycle at $20-26^{\circ} \mathrm{C}$ and $40-70 \%$ humidity, with ad libitum access to food and water. The wastes of the animal experiments were disposed of according to the Experimental Animal Guidelines of the Health Ministry of the People's Republic of China.

Grouping, modeling, and interventions. Three-day-old SD rats were randomly divided into the following six groups: i) The normal control (NC) group $(\mathrm{n}=21)$; ii) the hypoxic-ischemic (HI) group $(n=21)$; iii) the anemia group $(n=21)$; iv) the $\mathrm{HI}+$ anemia group $(\mathrm{n}=21)$; v) the early iron $(35 \mathrm{mg} / \mathrm{kg}$ once daily; Niferex; Schwarz Pharma, Inc., Zhuhai, China) treatment group $(n=3)$; and vi) the late iron treatment group $(n=3)$. The NC group underwent normal feeding. Rats in the HI group underwent bilateral common carotid artery ligation following the intraperitoneal injection of $10 \%$ chloral hydrate $(0.5 \mathrm{ml} / \mathrm{kg}$; Meilun Biology Technology Co., Dailan, China). The skin incisions were sutured, and the rats were allowed to recover for $1 \mathrm{~h}$, prior to placement in a sealed water bath containing a hypoxic gas mixture ( $92 \%$ nitrogen, $8 \%$ oxygen) at $37^{\circ} \mathrm{C}$ for $90 \mathrm{~min}$. In order to establish the anemia group, a 1-ml syringe needle was inserted at the strongest beating points of the heart at depth $\sim 0.5 \mathrm{~cm}$, and was used to take $0.4-0.5 \mathrm{ml}$ blood, and was pressed to stop the bleeding. Rats in the $\mathrm{HI}+$ anemia group were intraperitoneally injected with $10 \%$ chloral hydrate $(0.5 \mathrm{ml} / \mathrm{kg})$ and underwent the same surgical procedure and hypoxic exposure as the HI group, after which they received the same treatment as the anemia group. In the early and late iron treatment groups, rats commenced treatment with iron (35 mg/kg body weight) on days 1 and 7, respectively, following $\mathrm{HI}+$ anemia modeling. Treatments lasted until the end of the 28-day experimental period.

Tissue specimen preparation. Tissues were collected from 4- and 6-day-old rats for hematoxylin and eosin (H\&E) staining, from 28-day-old rats for electron microscopic examination, and 4-day-old rats for iron content and reverse transcription-quantitative polymerase chain reaction (RT-qPCR) measurements. Rats were anesthetized by the intraperitoneal injection of $10 \%$ chloral hydrate anesthesia $(0.5 \mathrm{ml} / \mathrm{kg})$, and then sacrificed by decapitation. Blood tests were conducted immediately, following the collection of blood in an anticoagulation tube. Five rats with hemoglobin levels $>100$ g/l, tested using Horiba ABX Pentra DF-120 Analyzer (Horiba Medical, Montpellier, France), were excluded from the anemia and $\mathrm{HI}+$ anemia groups.

Rat brain tissues were harvested under strictly aseptic conditions. The leptomeningeal vascular surface was completely stripped, removed and preserved in neutral formalin. Subsequently, the tissues were sliced into $14-\mu \mathrm{m}$ sections for H\&E staining, frozen and stored in a refrigerator at $-80^{\circ} \mathrm{C}$ for iron content and RT-qPCR measurements. They were placed in $2.5 \%$ formaldehyde amyl fixative (CellChip Biotechnology Co., Ltd., Beijing, China) for further processing by electron microscopy.

$H \& E$ staining. Brain tissues were placed in $30 \%$ sucrose overnight at $-4^{\circ} \mathrm{C}$. The tissue sections were stained with $10 \%$ hematoxylin dye for $3 \mathrm{~min}$, differentiated with $0.5 \%$ ethanol hydrochloride for several seconds and recovered with lithium carbonate saturated solution, prior to washing with distilled water. Subsequently, the tissue sections were stained with $0.5 \%$ eosin for $1 \mathrm{~min}$, dehydrated with gradient ethanol after washing, immersed in dimethyl benzene and mounted with neutral gum.

Detection of iron content in the brain. The brain tissues of rats in the NC, HI, anemia and HI + anemia groups were weighed and washed with phosphate-buffered saline. The tissue $(0.5 \times 0.5 \mathrm{~cm})$ was then mixed with homogenate buffer (Thermo Fisher Scientific, Inc., Waltham, MA, USA) to a volume ratio of 1:5, and homogenized at $400 \mathrm{x}$ g for $10 \mathrm{sec}, 6$ times. Then, the homogenates was centrifuged at $1,600 \mathrm{x}$ for $15 \mathrm{~min}$ at $4^{\circ} \mathrm{C}$, and the supernatant was separated. Brain homogenates were treated with $\mathrm{HNO}_{3}-\mathrm{H}_{2} \mathrm{O}_{2}$ solution and boiled until transformed into a colorless transparent liquid. The iron content in the brain tissue was quantified using atomic absorption spectrophotometry (14).

Immunofluorescence staining of oligodendrocytes. Brain tissue was immersed in fixative (4\% paraformaldehyde), dehydrated and embedded with optimal cutting temperature compound. The sections of rat brain tissue were preserved at $-4^{\circ} \mathrm{C}$. Then, sections were incubated with polyclonal primary rabbit anti-carbonic anhydrase II (CAII) antibody (1:200; Santa Cruz Biotechnology, Inc., Dallas, TX, USA) at $4^{\circ} \mathrm{C}$ overnight followed by incubation with the DyLight 594-labeled goat secondary antibody (1:500; ZSGB-Bio, Beijing, China) at room temperature for $30 \mathrm{~min}$. Cell nuclei were stained with Hoechst 33342 (ZSGB-Bio). The survival of oligodendrocytes around the ventricles was observed visually using an Olympus BX51 fluorescence microscope (Olympus Corporation, Tokyo, Japan).

Quantification of $m R N A$ expression levels of iron regulatory protein 2 (IRP2) and transferrin receptor (TFR) in rat brains. Total RNA was extracted from the brain tissue of the SD rats using TRIzol reagent (Abcam, Beijing, China) and treated with 
DNase I (cat. no. EN0521; Fermentas; Thermo Fisher Scientific, Inc.). The RNA product was then examined by agarose gel electrophoresis. Reverse transcription was performed to transcribe the total RNA into cDNA, using a Thermo First cDNA Synthesis kit (cat. no. 33-20102; SinoGene Scientific Co., Ltd., Beijing, China). The reaction system consisted of $10 \mu \mathrm{l}$ DNase, $1 \mu \mathrm{l}$ random primer, $4 \mu \mathrm{l} 5 \mathrm{X}$ reaction buffer, $2 \mu \mathrm{l}$ reverse transcriptase enzyme, and diethylpyrocarbonate water to a total volume of $20 \mu \mathrm{l}$. qPCR reactions were carried out using 2X SG Green qPCR mix (with ROX; cat. no. 22-10102, SinoGene Scientific Co., Ltd.) with a StepOnePlus Real-Time PCR system (Applied Biosystems; Thermo Fisher Scientific, Inc., Waltham, MA, USA). The primer sequences were designed using Primer Premier 5 software (Premier Biosoft International, Palo Alto, CA, USA) and were as follows: IRP2 forward, TTGTCCATGTTTAAAGCACTGA and reverse, ACACTGAATCTGGAGCGTCTA (product length, 82 bp); TFR forward, TGGACTGCAGGAGACTAT and reverse, GTACCCAGGACGACTTTAT (product length, 116 bp); $\beta$-actin forward, CGTTGACATCCGTAAAGACC and reverse, CTAGGAGCCAGAGCAGTAATC (product length 116 bp). $\beta$-actin was used as a reference gene. qPCR reactions were performed under the following conditions: $10 \mathrm{~min}$ at $95^{\circ} \mathrm{C}$, followed by 40 cycles of $15 \mathrm{sec}$ at $95^{\circ} \mathrm{C}, 15 \mathrm{sec}$ at $53^{\circ} \mathrm{C}$ and $35 \mathrm{sec}$ at $72^{\circ} \mathrm{C}$. An additional step was used $\left(95^{\circ} \mathrm{C}\right.$ for $15 \mathrm{sec}, 60^{\circ} \mathrm{C}$ for $30 \mathrm{sec}$ and $95^{\circ} \mathrm{C}$ for $15 \mathrm{sec}$ ) for dissociation curve analysis. Data were analyzed using the $2^{-\Delta \Delta C t}$ method (15).

Electron microscopy. Three 28-day-old newborn rats were selected from each group and were sacrificed by decapitation, prior to extraction of the periventricular tissues. The tissues were cut into $1-\mathrm{mm}^{3}$ samples and immersed in a solution containing $2.0 \%$ paraformaldehyde and $2.5 \%$ glutaraldehyde, followed by fixing with $1 \%$ osmium tetroxide for $2 \mathrm{~h}$ at $4^{\circ} \mathrm{C}$. Subsequently, the tissue sections were washed three times with $0.2 \mathrm{M}$ sodium cacodylate buffer for $10 \mathrm{~min}$, and then three times with double distilled water for $10 \mathrm{~min}$, followed by dehydration using alcohol gradients. The samples were further embedded in epoxy resin (Zhongke Chemical Co., Ltd., Tianjin, China), cut into $100 \mathrm{~nm}$ slices, stained with uranyl acetate and lead citrate, and observed under an H-7650 Transmission Electron Microscope (Hitachi, Ltd., Tokyo, Japan). The brain tissue ultrastructure was observed under visual observation at x20,000 magnification, and the number of myelin sheaths was counted under x 5,000 magnification; five fields of view were randomly selected for each slice.

Statistical analysis. The results from each group were compared using multivariate analysis of variance. Comparisons between two groups were performed using the independent samples t-test. Correlation analysis between two variables was conducted using linear regression analysis. Statistical analyses were conducted using SPSS software, version 13.0 (SPSS, Inc., Chicago, IL, USA). $\mathrm{P}<0.05$ was considered to indicate a statistically significant difference.

\section{Results}

Establishment of a rat model of anemia. A rat model of anemia was established using immature newborn rats. The mean
Table I. Hemoglobin content of each group.

\begin{tabular}{lc}
\hline Group & Hemoglobin $(\mathrm{g} / \mathrm{l})$ \\
\hline NC group & $141.75 \pm 6.54$ \\
Anemia group & $79.25 \pm 9.49^{\mathrm{a}}$ \\
HI group & $150.12 \pm 12.19$ \\
HI + anemia group & $84.88 \pm 8.34^{\mathrm{a}}$
\end{tabular}

Data are presented as the mean \pm standard deviation ( $\mathrm{n}=8$ per group). ${ }^{\mathrm{a}} \mathrm{P}<0.01$ vs. the $\mathrm{NC}$ group. $\mathrm{F}=125.548 ; \mathrm{P}<0.001$ among the groups. $\mathrm{NC}$, normal control; HI, hypoxic-ischemic.

Table II. Iron content of each group.

\begin{tabular}{ll}
\hline Group & Iron $(\mu \mathrm{g} / \mathrm{g})$ \\
\hline NC group & $17.45 \pm 1.21$ \\
Anemia group & $14.29 \pm 0.97^{\mathrm{a}, \mathrm{b}}$ \\
HI group & $20.62 \pm 5.11^{\mathrm{a}, \mathrm{c}}$ \\
HI + anemia group & $16.99 \pm 2.89$
\end{tabular}

Data are presented as the mean \pm standard deviation ( $\mathrm{n}=8$ per group). ${ }^{\mathrm{a}} \mathrm{P}<0.05$ vs. the $\mathrm{NC}$ group; ${ }^{\mathrm{b}} \mathrm{P}<0.05$ vs. the $\mathrm{HI}$ group; ${ }^{\mathrm{c}} \mathrm{P}<0.05$ vs. the $\mathrm{HI}+$ anemia group. $\mathrm{F}=5.853 ; \mathrm{P}=0.003$ among the groups. $\mathrm{NC}$, normal control; HI, hypoxic-ischemic.

hemoglobin content of each group is presented in Table I. The hemoglobin contents differed significantly among the groups $(\mathrm{P}<0.001)$; the levels in the anemia and $\mathrm{HI}+$ anemia groups were lower than those in the NC and HI groups, and met the standards of the anemia model, since they were $\leq 100 \mathrm{~g} / \mathrm{l}$.

$H \& E$ staining of the rat brain tissue sections. In the NC group, the brain tissues looked healthy, with neatly ordered, normally shaped cells with a central nucleus and clearly visible nucleoli (Fig. 1). However, in the HI group, a large number of cells in the cortical, hippocampal and periventricular areas exhibited necrosis, vacuolar degeneration, nuclear condensation and fragmentation. In some cases, dissolution of the nucleus was observed.

Brain iron content. As shown in Table II, there were significant differences in brain iron content among the groups $(\mathrm{F}=5.853$; $\mathrm{P}=0.003)$. Further analysis indicated that the iron content in the anemia group was significantly lower than that in the $\mathrm{NC}$ group ( $\mathrm{P}=0.047)$; whereas the iron content of the HI group was significantly higher compared with that in the NC group $(\mathrm{P}=0.045)$. No significant differences in iron content were observed between the $\mathrm{HI}+$ anemia group and the NC group $(\mathrm{P}=0.766)$. The iron content of the HI group was significantly higher than that of the $\mathrm{HI}+$ anemia group $(\mathrm{P}=0.024)$.

Immunofluorescence of oligodendrocytes. As depicted in Fig. 2, the number of oligodendrocytes around the ventricles in the HI group was markedly decreased compared with that in the $\mathrm{NC}$ group. Furthermore, the number of viable oligodendrocyte 


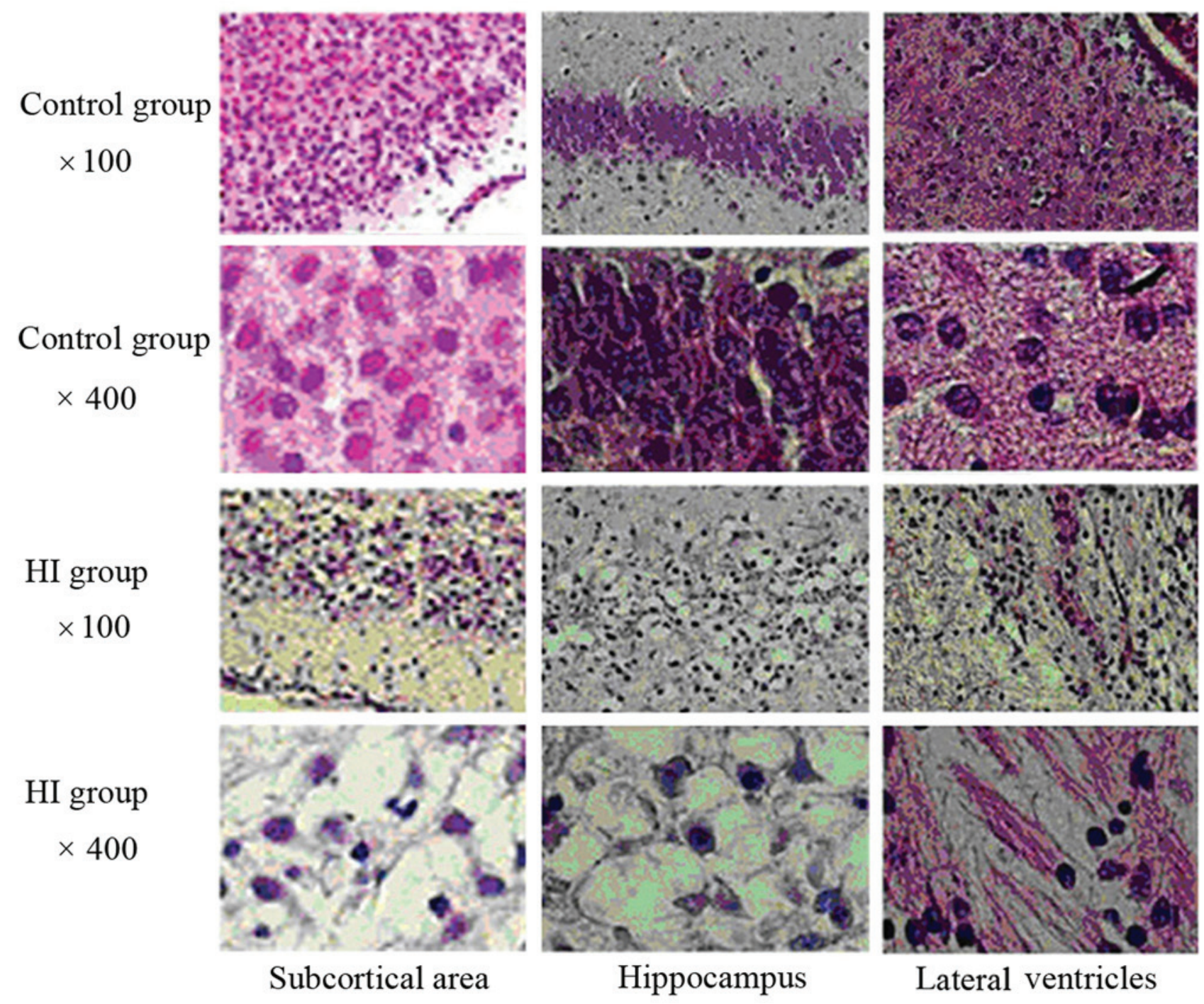

Figure 1. Hematoxylin and eosin staining of the rat brain cortex, hippocampus and areas surrounding the lateral ventricles in the normal control and hypoxic-ischemic (HI) groups at x100 and x400 magnification.

glial cells in the ventricles of the anemia group was slightly increased compared with that in the NC group, and the number of viable oligodendrocytes in the $\mathrm{HI}+$ anemia group was increased compared with that in the HI group.

mRNA expression levels of IRP 2 and TFR in the rat brains. As shown in Tables III and IV, there were significant differences in the mRNA expression levels of IRP2 and TFR in the rat brains among the groups (IRP2: $\mathrm{F}=7.220, \mathrm{P}=0.003$; TFR: $\mathrm{F}=5.169$, $\mathrm{P}=0.011)$. Further analysis suggested that the mRNA expression levels of IRP2 were significantly decreased in the anemia group compared with those in the $\mathrm{NC}$ group $(\mathrm{P}=0.05)$, and that they were significantly increased in the HI group $(\mathrm{P}=0.023)$ compared with the NC group. There was no significant difference in IRP 2 mRNA expression between the $\mathrm{HI}+$ anemia and $\mathrm{NC}$ groups $(\mathrm{P}=0.851)$. The mRNA expression levels of IRP2 were significantly increased in the HI group compared with those in the $\mathrm{HI}+$ anemia group $(\mathrm{P}=0.033)$. The changes in the mRNA expression levels of TFR were similar to those of the mRNA expression levels of IRP2 in each group. The mRNA expression levels of TFR in the anemia group were markedly lower than those in the normal control group, but were not significantly different $(\mathrm{P}=0.128)$. The mRNA expression levels of TFR were significantly higher in the HI group than in the $\mathrm{NC}$ group $(\mathrm{P}=0.041)$ and the $\mathrm{HI}+$ anemia group $(\mathrm{P}=0.016)$. There was no significant difference between the $\mathrm{HI}+$ anemia and $\mathrm{NC}$ groups $(\mathrm{P}=0.643)$.

Electron microscopy results. In the rat brains of the NC group a uniform thickness and density, as well as a regular shape and clear boundaries of the myelin sheath, were observed in the intact structure. Furthermore, the nerve cells had a refined structure, the organelles in the cytoplasm appeared to have a normal morphology, the mitochondrial structure was intact, the distribution of chromatin in the nucleus was uniform, the axonal microtubules and filaments were well-organized and integrity of structure was detected. In addition, no pathological changes were observed in the surrounding areas of the brain (Fig. 3A and B).

The number of myelin sheaths in the rat brains of the HI group was significantly reduced compared with the number in the NC group. Furthermore, incomplete structures, irregular 
A

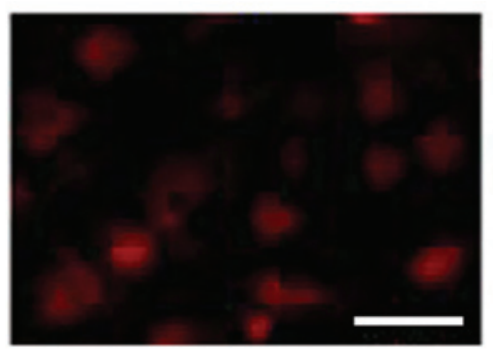

B

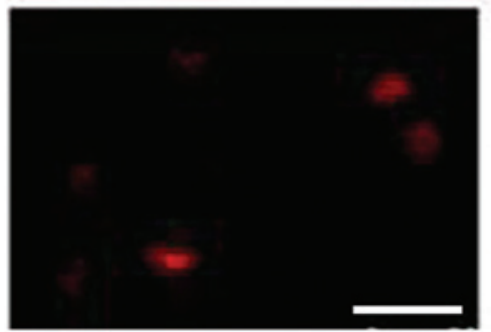

C

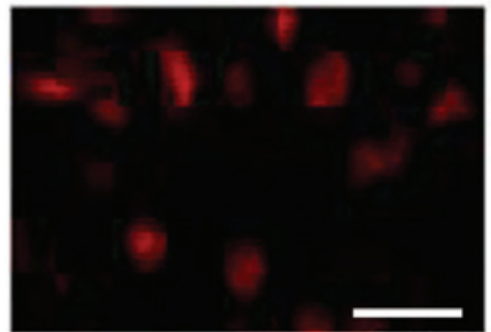

D

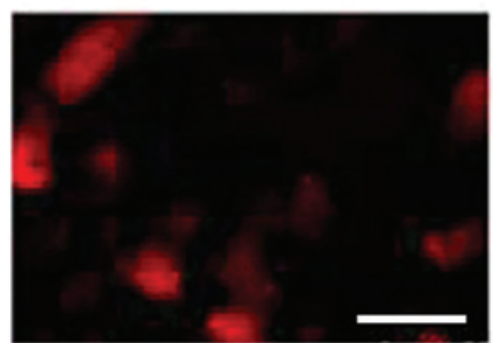

CA II
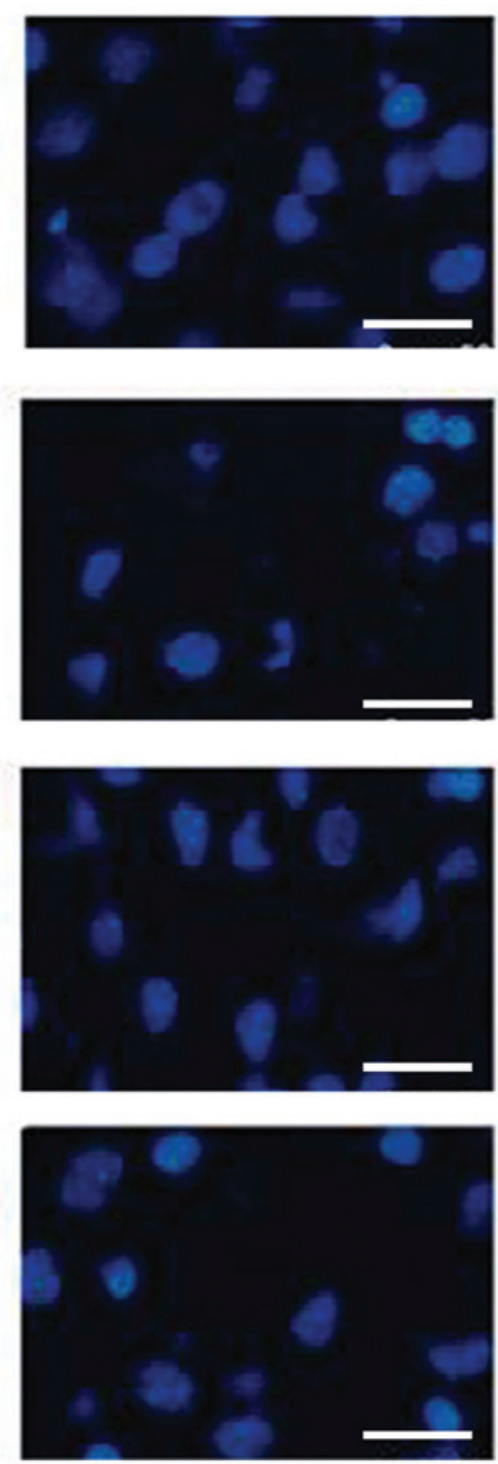

Nucleus
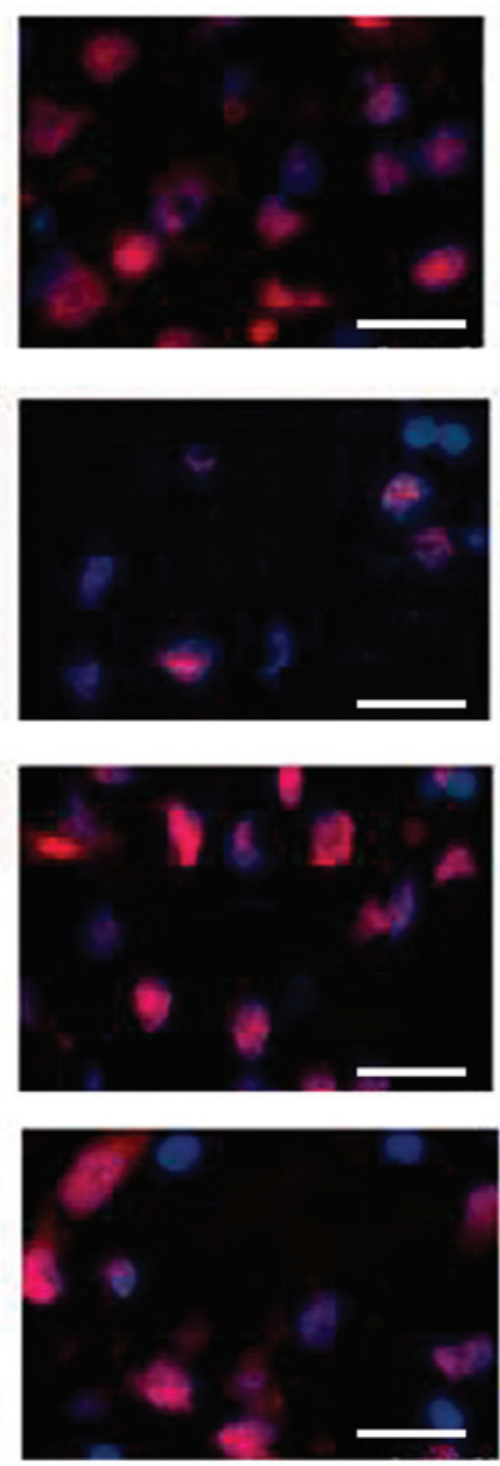

Merge

Figure 2. Immunofluorescence of oligodendrocytes. (A) In the normal control (NC) group, numerous oligodendrocytes were detected around the ventricles of the rats. (B) In the hypoxic-ischemic (HI) group, a significant reduction in the number of oligodendrocytes was observed compared with that in the NC group. (C) In the anemia group, only a slight reduction in the number of oligodendrocytes was observed compared with that in the NC group. (D) In the $\mathrm{HI}+$ anemia group, the number of oligodendrocytes was increased compared with that in the HI group. Hoechst 33342 staining. Scale bar, $50 \mu \mathrm{m}$. CA II, carbonic anhydrase II.

organelle morphologies and a disrupted arrangement were observed. Edema was evident in the cytoplasmic organelles of the neurons, including a reduced number of mitochondria in comparison with the NC group. Similar results were observed for other characteristics, including edema morphology, ridge breaks and even disappearance, severe vacuolization of some mitochondria, chromatin condensation in the nucleus, disappearance of the cytoplasm and nucleus, swelling of the surrounding nerve fibers and the fracture and reduction of axonal microtubules and filaments (Fig. 3C and D).

The number of myelin sheaths was markedly decreased in the anemia group compared with the NC group. Furthermore, partial incomplete structures and irregular morphology were observed in the anemia group. Slight edema was detected in the cytoplasmic organelles of some nerve cells, the number of mitochondria was decreased, the ridge structure was partially broken, and there was an even distribution of chromatin in the nucleus. The filaments, including the microtubules, in axons were well arranged and their structures appeared to be intact (Figs. 4-6).

As compared with the HI group, the number of myelin sheaths was markedly increased in the $\mathrm{HI}+$ anemia group, and this was associated with structural improvements. Extended edema of the neuron organelles and mitochondria was alleviated. In addition, the ridge structure was well maintained and chromatin condensation in the nucleus was markedly reduced. Similar to the HI group, only slight breaks were observed in the filaments and microtubules of the axons in the HI + anemia group (Figs. 4-6).

As compared with the $\mathrm{HI}+$ anemia group, the number of myelin sheaths was markedly reduced in the early iron treatment group. This was accompanied by structural disorder, 
Table III. mRNA expression levels of IRP2 in each group (relative levels vs. $\beta$-actin).

\begin{tabular}{ll}
\hline Group & IRP2 \\
\hline NC group & $1.11 \pm 0.13$ \\
Anemia group & $0.87 \pm 0.25^{\mathrm{a}, \mathrm{b}}$ \\
HI group & $1.40 \pm 0.17^{\mathrm{a}, \mathrm{c}}$ \\
HI + anemia group & $1.13 \pm 0.15$ \\
\hline
\end{tabular}

Data are presented as the mean \pm standard deviation ( $\mathrm{n}=5$ per group). ${ }^{\mathrm{a}} \mathrm{P}<0.05$ vs. the $\mathrm{NC}$ group; ${ }^{\mathrm{b}} \mathrm{P}<0.01$ vs. the $\mathrm{HI}$ group; ${ }^{\mathrm{c}} \mathrm{P}<0.05$ vs. the $\mathrm{HI}+$ anemia group. $\mathrm{F}=7.220 ; \mathrm{P}=0.003$ among the groups. IRP2, iron regulatory protein 2 ; $\mathrm{NC}$, normal control; HI, hypoxic-ischemic.

Table IV. mRNA expression levels of TFR in each group (relative levels vs. $\beta$-actin).

\begin{tabular}{ll}
\hline Group & TFR \\
\hline NC group & $1.11 \pm 0.19$ \\
Anemia group & $0.92 \pm 0.27^{\mathrm{a}, \mathrm{b}}$ \\
HI group & $1.37 \pm 0.12^{\mathrm{b}, \mathrm{c}}$ \\
HI + anemia group & $1.05 \pm 0.24$
\end{tabular}

Data are presented as the mean \pm standard deviation ( $\mathrm{n}=5$ per group). ${ }^{\mathrm{a}} \mathrm{P}<0.01$ vs. the $\mathrm{HI}$ group; ${ }^{\mathrm{b}} \mathrm{P}<0.05$ vs. the $\mathrm{HI}+$ anemia group; ${ }^{\mathrm{C}} \mathrm{P}<0.05$ vs. the NC group. $\mathrm{F}=5.169 ; \mathrm{P}=0.011$ among the groups. TFR, transferrin receptor; $\mathrm{NC}$, normal control; $\mathrm{HI}$, hypoxic-ischemic.

partial fracture of myelin sheaths and a disordered arrangement. Nerve cell edema was largely aggregated, with marked reductions in the number of mitochondria and vacuoles. Furthermore, chromatin condensation and shrinkage of the nucleus were detected in the nerve cells. Similar to the HI group, more severe symptoms were observed in the early iron treatment group, including breakages and a reduced number of filaments and microtubules in axons, when compared with the $\mathrm{HI}+$ anemia group (Figs. 4-6).

The number of myelin sheaths was markedly increased and the structure was improved in the late iron treatment group compared with the HI + anemia group. Alleviation of edema was detected in the intracellular organelles and mitochondria, and the ridge structure was well maintained and chromatin condensation in the nucleus of nerve cells was markedly reduced. However, similar to the observations for the HI group, there was a significant improvement in the damaged filaments and microtubules of the axon, which were decreased in number (Figs. 4-6).

As shown in Table $\mathrm{V}$, there was a significant difference in the number of myelin sheaths among the different groups, when observed by low-resolution electron microscopy $(\mathrm{F}=118.429, \mathrm{P}<0.001)$. Further analysis indicated that the number of myelin sheaths in the $\mathrm{HI}+$ anemia group and the HI group were markedly lower than the number in the NC group $(\mathrm{t}=12.691, \mathrm{P}<0.001 ; \mathrm{t}=6.091, \mathrm{P}=0.001$, respectively). The number of myelin sheaths in the early iron treatment
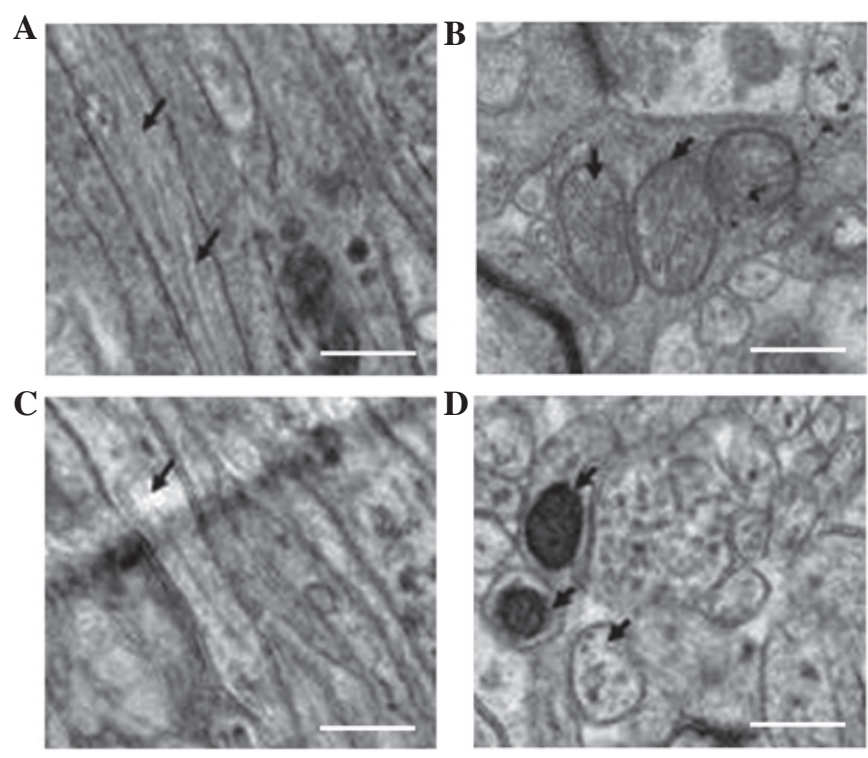

Figure 3. Electron microscope snapshots of the axons, myelin and mitochondria in the rat brains (magnification, $x 20,000$ ). (A) In the normal control (NC) group, normal axons were observed. The arrows point to the axon filament microtubules. (B) Mitochondria in the NC group: The arrows indicate the ridge structure in the mitochondria, which exhibit a clear and complete shape. (C) In the hypoxic-ischemic (HI) group, the axonal microtubules and filaments were fractured and reduced in number (arrows). (D) Mitochondria in the HI group: The arrows indicate an increase in electron density, fractionation and disappearance of the ridges in the mitochondria. Scale bar, $1 \mu \mathrm{m}$.

group was significantly reduced compared with that in the $\mathrm{HI}+$ anemia group $(\mathrm{t}=10.928, \mathrm{P}<0.001)$. The number of myelin sheaths in the late iron treatment group was significantly lower than that in the $\mathrm{NC}$ group $(\mathrm{t}=8.356, \mathrm{P}<0.001)$; however, it was significantly higher than that in the $\mathrm{HI}+$ anemia group $(\mathrm{t}=4.811, \mathrm{P}=0.003)$. As compared with the HI group, a higher number of myelin sheaths was observed in the HI + anemia group ( $\mathrm{t}=2.524, \mathrm{P}=0.045)$, although the number of myelin sheaths in these two groups was significantly lower than the number in the NC group $(\mathrm{t}=12.277, \mathrm{P}<0.001 ; \mathrm{t}=12.691$, $\mathrm{P}<0.001$, respectively).

\section{Discussion}

The production, differentiation and maturation of oligodendrocyte precursor cells differ between humans and rats; in particular, human oligodendrocyte precursor cells arise during early pregnancy ( $<32$ weeks) and gradually differentiate into mature oligodendrocytes. In addition, the fastest period of axon myelination occurs during the first year after birth. Conversely, rat oligodendrocyte precursor cells increase in numbers during the first week post-birth, and the myelin sheaths are formed during the first 2 weeks, with the fastest period of myelin formation occurring during the first 3 weeks after birth (16). The nervous system of a 7-day-old newborn rat is equivalent to that at 32 weeks of human pregnancy, and that of a 2-4-day-old newborn rat is similar to that of a human at middle pregnancy (28-31 weeks) (16). Therefore, the 3-day-old newborn rat model of HI-induced brain injury established in the present study is suitable for use as a reference for pre-term infants with HI-induced brain injury that can be used for the investigation of potential clinical treatments. 

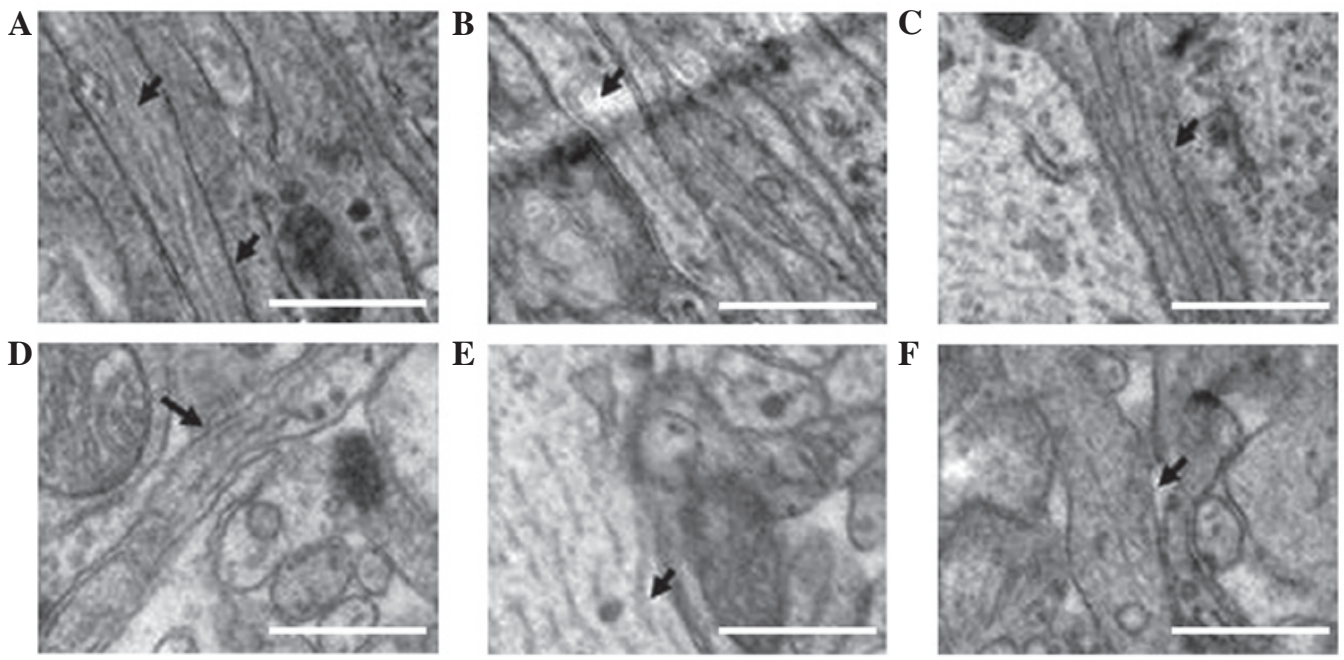

Figure 4. Electron microscope images of the filaments and microtubules in each group (magnification, $\mathrm{x} 50,000$ ). (A) In the normal control group, the axonal microtubules and filaments were well-arranged and structured. (B) In the hypoxic-ischemic (HI) group, axonal microtubules and filaments were disrupted and decreased in number. (C) In the anemia group, axonal microtubules and filaments were well arranged and structured. (D) The ultrastructure of the HI + anemia group was comparable to that of the HI group. (E) In the early iron treatment group, the structural integrity was worse than that in the HI group and (F) in the late iron treatment group, the structure was improved compared with that in the HI group. The arrows indicate filaments and microtubules. Scale bar, $1 \mu \mathrm{m}$
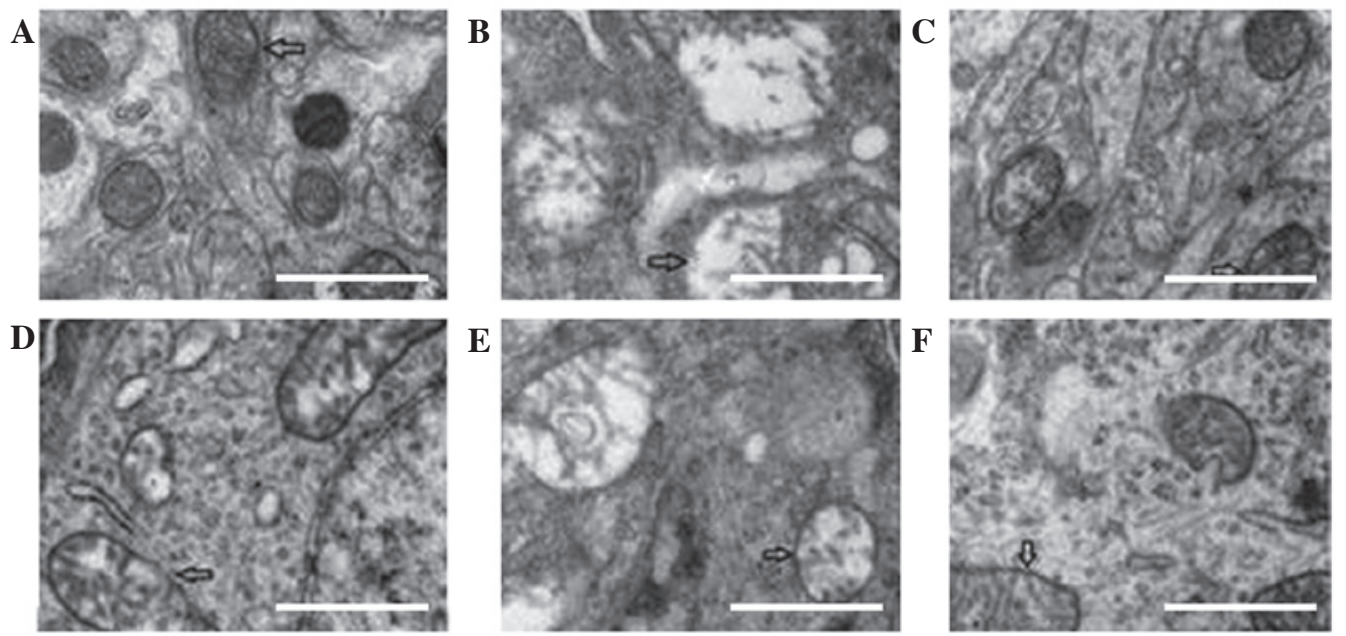

Figure 5. Electron microscope snapshots of mitochondrial structural changes in the (A) normal control, (B) hypoxic-ischemic (HI), (C) anemia, (D) HI + anemia, (E) early iron treatment and (F) late iron treatment groups (magnification, x50,000; scale bar, $1 \mu \mathrm{m}$ ).
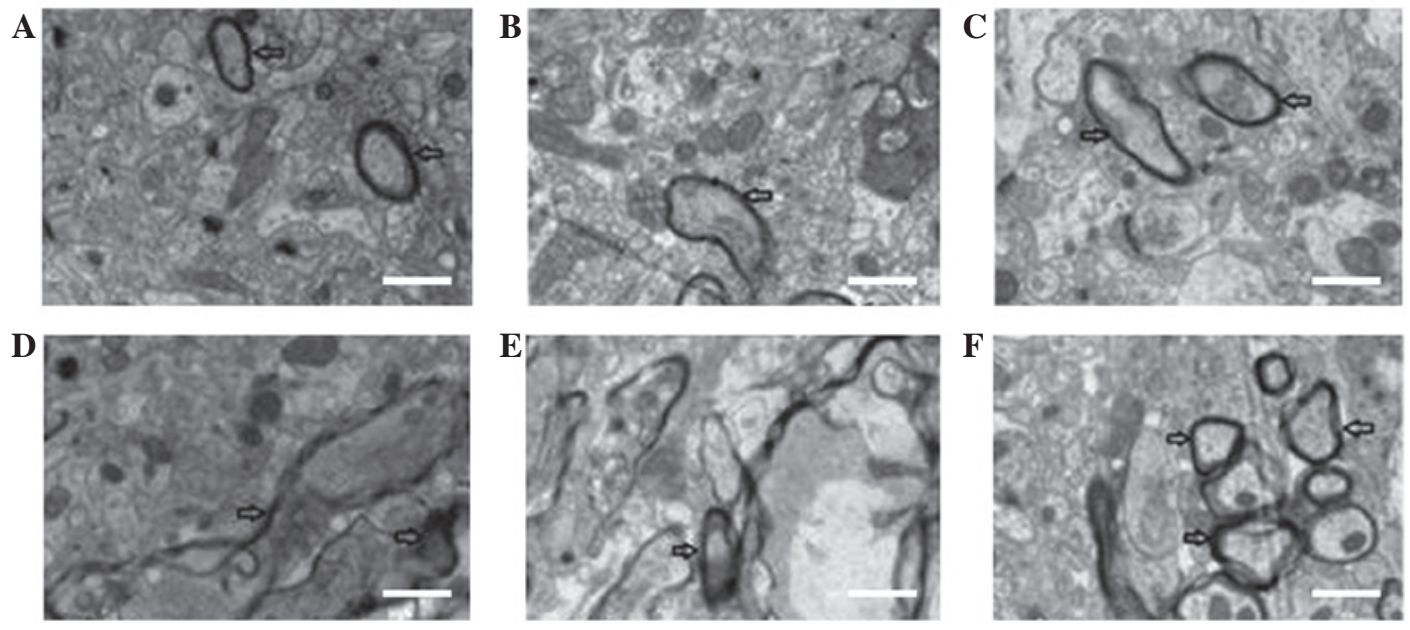

Figure 6. Electron microscope snapshots of the areas surrounding the lateral brain ventricles in the (A) normal control, (B) hypoxic-ischemic (HI), (C) anemia, (D) $\mathrm{HI}+$ anemia, (E) early iron treatment and (F) late iron treatment groups (magnification, x20,000; scale bar, $1 \mu \mathrm{m}$ ). 
Table V. Number of myelin sheaths in each group.

\begin{tabular}{ll}
\hline Group & Myelin count \\
\hline NC group & $75.00 \pm 4.55$ \\
HI group & $38.00 \pm 3.65^{\mathrm{a}}$ \\
Anemia group & $58.25 \pm 3.09^{\mathrm{a}}$ \\
HI + anemia group & $43.50 \pm 2.38^{\mathrm{a}, \mathrm{b}}$ \\
Early iron treatment & $21.25 \pm 3.30^{\mathrm{a}, \mathrm{c}}$ \\
Late iron treatment & $52.50 \pm 2.88^{\mathrm{a}, \mathrm{c}}$
\end{tabular}

Data are presented as the mean \pm standard deviation $(n=3)$. The number of myelin sheaths in each group were determined using electron microscopy. ${ }^{\mathrm{a}} \mathrm{P}<0.01$ vs. the $\mathrm{NC}$ group; ${ }^{\mathrm{b}} \mathrm{P}<0.01$ vs. the $\mathrm{HI}$ group; ${ }^{c} \mathrm{P}<0.05$ vs. the $\mathrm{HI}+$ anemia group. $\mathrm{F}=118.429 ; \mathrm{P}<0.001$. NC, normal control; HI, hypoxic-ischemic.

The results of the present study suggested that HI may lead to an increased postnatal brain iron content. Under an electron microscope, an incomplete structure, loosely arranged cells, oligodendrocyte and mitochondria abnormalities, myelin damage, lamellar separation and a reduced number of myelin sheaths were observed in the HI group, as compared with 28-day-old rats in the NC group. These results indicated that the early occurrence of HI in immature rats may affect the development of the nervous system; the time following birth for immature rats is a critical period for oligodendrocyte and myelin development, which causes oligodendrocyte precursor cells to be highly sensitive to the effects of adverse factors, such as hypoxia (17). Therefore, myelin damage is more severe at this time.

When HI was accompanied by anemia, the iron content of brain tissues was significantly reduced compared with that in the HI group. Similar pathological changes were observed under the light microscope in the $\mathrm{HI}+$ anemia and HI groups. However, under the electron microscope, ultrastructural changes were significantly reduced in the HI + anemia group when compared with those in the HI group, and suggested that the number of myelin sheaths was significantly higher in the $\mathrm{HI}+$ anemia group compared with the HI group. These results suggested that anemia was able to reduce the iron content of the brain and concomitantly attenuate HI-induced brain injury. Therefore, early anemia may protect against HI-induced brain injury. However, significant ultrastructural changes were observed in both the HI + anemia and anemia groups after 28 days. In addition, the number of myelin sheaths, as detected using low-resolution electron microscopy, was markedly lower in the two groups, as compared with the NC group. Therefore, it may be beneficial to develop a novel intervention strategy for neonatal anemia, although the time point of intervention may influence the prognosis if the pre-term infant has suffered from HI-induced brain injury.

Considerable ultrastructural changes in the brains of the rats in the $\mathrm{HI}+$ anemia group, including changes within the myelin, synapses and mitochondria, were observed by electron microscopy. In the early iron treatment group, iron gavage was commenced 4 days after birth (1 day following the occurrence of $\mathrm{HI}$ and anemia), and continued until 28 days. When observed under an electron microscope, the number of myelin sheaths was found to be markedly lower in the early iron treatment group, as compared with that in the NC, HI + anemia, HI and anemia groups. These results suggested that iron treatment during the early postnatal stages may exacerbate brain injury or hinder the repair of brain damage. Therefore, in cases where HI-induced brain injury is accompanied by anemia in preterm children, the supplementation of iron during the early stages of development may not be beneficial.

In the HI-exposed immature rats that had anemia for a prolonged time period, such as until 10 days post-birth (7 days following the occurrence of $\mathrm{HI}$ and anemia) and were then treated with iron by gavage until 28 days (HI + anemia group, with late treatment), the brain injury was markedly alleviated when observed under an electron microscope. Beneficial effects of late iron treatment were observed in, for example, the ultrastructure of myelin, synapses and mitochondria.

The number of myelin sheaths was significantly higher in the late iron treatment group compared with the early iron treatment and HI + anemia groups. This suggested that late iron treatment may be beneficial for repairing the damaged brain, without incurring any adverse effects. Therefore, late iron treatment for HI-induced brain injury accompanied by anemia may facilitate the repair of brain injury.

There are two forms of IRP, namely IRP1 and IRP2 (18-20). When the existence of cytoplasmic RNA binding proteins, also known as IRPs, was discovered, it was demonstrated that the proteins were able to regulate the expression levels of ferritin and transferrin at the post-transcriptional level, thus suggesting that they may have an important role in regulating the cellular metabolism of iron $(21,22)$. Subsequently, IRP2 was discovered, and was shown to have a very similar structure and function to IRP1 (23-26). In cells, IRP1 is predominantly regulated by the intracellular iron concentration; when there is an iron deficiency, the binding affinity of IRP1 to the iron-responsive element (IRE) in ferritin RNA is increased, leading to the inhibition of ferritin mRNA translation. This in turn stabilizes the TFR mRNA, thereby enabling the cellular uptake of iron and preventing the occurrence of iron storage (27). Conversely, when the intracellular iron concentration is increased, the binding affinity of IRP1 to the IRE in ferritin mRNA is reduced, leading to the reduced cellular uptake of iron and an increased rate of iron storage (27). IRP2 is also regulated by the intracellular iron concentration, although by a different mechanism. When the intracellular iron concentration is increased, the binding affinity of IRP2 with mRNA decreases, which is accompanied by decreased levels of protein expression. This in turn results in iron-mediated protein hydrolysis (28). The results of the present study suggested that the mRNA expression levels of IRP2 in the rats of the HI model group was significantly increased as compared with that in the NC group, thus suggesting that HI may increase the mRNA expression levels of IRP2 in the brain, leading to the increased iron content associated with HI-induced brain damage.

In conclusion, anemia reduced the rate of increase in iron content in the brain tissues of hypoxic-ischemic brain-injured newborn immature rats. Iron supplementation during the early stage of newborn infancy may result in aggravation of brain injury or affect the recovery of brain damage. However, iron 
supplementation at a later stage after birth appears to result in a significant alleviation of brain injury.

\section{Acknowledgements}

This study was supported by grants from the National Natural Science Foundation of China (grant nos. 81370741 and 81401245), the National Natural Science Foundation of Beijing and Key Technology Program of Beijing Education Committee (grant no. KZ201410025025).

\section{References}

1. Rahman A, Abdellatif M, Sharef SW, Fazalullah M, Al-Senaidi K Khan AA, Ahmad M, Kripail M, Abuanza M and Bataclan F: Changing survival rate of infants born before 26 gestational weeks: Single-centre study. Sultan Qaboos Univ Med J 15: e351-e356, 2015

2. Olivieri I, Bova SM, Urgesi C, Ariaudo G, Perotto E, Fazzi E, Stronati M, Fabbro F, Balottin U and Orcesi S: Outcome of extremely low birth weight infants: What's new in the third millennium? Neuropsychological profiles at four years. Early Hum Dev 88: 241-250, 2012.

3. Skiöld B, Vollmer B, Böhm B, Hallberg B, Horsch S, Mosskin M, Lagercrantz H, Ådén U and Blennow M: Neonatal magnetic resonance imaging and outcome at age 30 months in extremely preterm infants. J Pediatr 160: 559-566, e1, 2012.

4. Madan N, Rusia U, Sikka M, Sharma S and Shankar N: Developmental and neurophysiologic deficits in iron deficiency in children. Indian J Pediatr 78: 58-64, 2011

5. Connor JR: Iron acquisition and expression of iron regulatory proteins in the developing brain: Manipulation by ethanol exposure, iron deprivation and cellular dysfunction. Dev Neurosci 16: 233-247, 1994.

6. Grantham-Mcgregor S and Baker-Henningham H: Iron deficiency in childhood: Causes and consequences for child development. Annales Nestlé 68: 105-119, 2010.

7. Ong WY, Tanaka K, Dawe GS, Ittner LM and Farooqui AA: Slow excitotoxicity in Alzheimer's disease. J Alzheimers Dis 35: 643-668, 2013.

8. Suttkus A, Rohn S, Jäger C, Arendt T and Morawski M Neuroprotection against iron-induced cell death by perineuronal nets - an in vivo analysis of oxidative stress. Am J Neurodegener Dis 1: 122-129, 2012.

9. Bartzokis G, Lu PH, Tishler TA, Fong SM, Oluwadara B, Finn JP, Huang D, Bordelon Y, Mintz J and Perlman S: Myelin breakdown and iron changes in Huntington's disease: Pathogenesis and treatment implications. Neurochem Res 32: 1655-1664, 2007.

10. Tanskanen M, Mäkelä M, Myllykangas L, Rastas S, Sulkava R and Paetau A: Intracerebral hemorrhage in the oldest old: A population-based study (Vantaa 85+). Front Neurol 3: 103, 2012.

11. Dong M, Xi G, Keep RF and Hua Y: Role of iron in brain lipocalin 2 upregulation after intracerebral hemorrhage in rats. Brain Res 1505: 86-92, 2013.
12. Guo C, Wang P, Zhong ML, Wang T, Huang XS, Li JY and Wang ZY: Deferoxamine inhibits iron induced hippocampal tau phosphorylation in the Alzheimer transgenic mouse brain. Neurochem Int 62: 165-172, 2013.

13. Kostic M, Zivkovic N and Stojanovic I: Multiple sclerosis and glutamate excitotoxicity. Rev Neurosci 24: 71-88, 2013.

14. Fitsanakis V A, Zhang N, Anderson J G, Erikson KM, Avison MJ, Gore JC and Aschner M: Measuring brain manganese and iron accumulation in rats following 14 weeks of low-dose manganese treatment using atomic absorption spectroscopy and magnetic resonance imaging. Toxicol Sci 103: 116-124, 2008.

15. Arocho A, Chen B, Ladanyi M and Pan Q: Validation of the 2-DeltaDeltaCt calculation as an alternate method of data analysis for quantitative PCR of BCR-ABL P210 transcripts. Diagn Mol Pathol 15: 56-61, 2006.

16. Back SA, Luo NL, Borenstein NS, Levine JM, Volpe JJ and Kinney HC: Late oligodendrocyte progenitors coincide with the developmental window of vulnerability for human perinatal white matter injury. J Neurosci 21: 1302-1312, 2001.

17. Khwaja $\mathrm{O}$ and Volpe JJ: Pathogenesis of cerebral white matter injury of prematurity. Arch Dis Child-Fetal Neonatal Ed 93: F153-F161, 2008.

18. Muckenthaler MU, Galy B and Hentze MW: Systemic iron homeostasis and the iron-responsive element/iron-regulatory protein (IRE/IRP) regulatory network. Annu Rev Nutr 28: 197-213, 2008

19. Wallander ML, Leibold EA and Eisenstein RS: Molecular control of vertebrate iron homeostasis by iron regulatory proteins. Biochim Biophys Acta 1763: 668-689, 2006.

20. Ganz T: Hepcidin and iron regulation, 10 years later. Blood 117: 4425-4433, 2011.

21. Davis $M$ and Clarke S: Influence of microRNA on the maintenance of human iron metabolism. Nutrients 5: 2611-2628, 2013.

22. Theil EC: Ferritin: The protein nanocage and iron biomineral in health and in disease. Inorg Chem 52: 12223-12233, 2013

23. Sanchez M, Galy B, Schwanhaeusser B, Blake J, Bähr-Ivacevic T, Benes V, Selbach M, Muckenthaler MU and Hentze MW: Iron regulatory protein-1 and-2: Transcriptome-wide definition of binding mRNAs and shaping of the cellular proteome by iron regulatory proteins. Blood 118: e168-e179, 2011.

24. Shpyleva SI, Tryndyak VP, Kovalchuk O, Starlard-Davenport A, Chekhun VF, Beland FA and Pogribny IP: Role of ferritin alterations in human breast cancer cells. Breast Cancer Res Treat 126: 63-71, 2011

25. Wang W, Deng Z, Hatcher H, Miller LD, Di X, Tesfay L, Sui G, D'Agostino RB Jr, Torti FM and Torti SV: IRP2 regulates breast tumor growth. Cancer Res 74: 497-507, 2014.

26. do Nascimento PR, Martins DR, Monteiro GR, Queiroz PV, Freire-Neto FP, Queiroz JW, Morais Lima AL and Jeronimo SM: Association of pro-inflammatory cytokines and iron regulatory protein 2 (IRP2) with Leishmania burden in canine visceral leishmaniasis. PLOS One 8: e73873, 2013.

27. Khan MA, Ma J, Walden WE, Merrick WC, Theil EC and Goss DJ: Rapid kinetics of iron responsive element (IRE) RNA/iron regulatory protein 1 and IRE-RNA/eIF4F complexes respond differently to metal ions. Nucleic Acids Res 42: 6567-6577, 2014.

28. Hausmann A, Lee J and Pantopoulos K: Redox control of iron regulatory protein 2 stability. FEBS Lett 585: 687-692, 2011. 